\title{
Japanese Adverse Reaction Terminology
}

National Cancer Institute

\section{Source}

National Cancer Institute. Japanese Adverse Reaction Terminology. NCI Thesaurus. Code C71885.

An adverse reaction terminology created in Japan, based on the World Health Organization's Adverse Reaction Terminology. 